International Journal of Scholarly Papers for Media and Communications

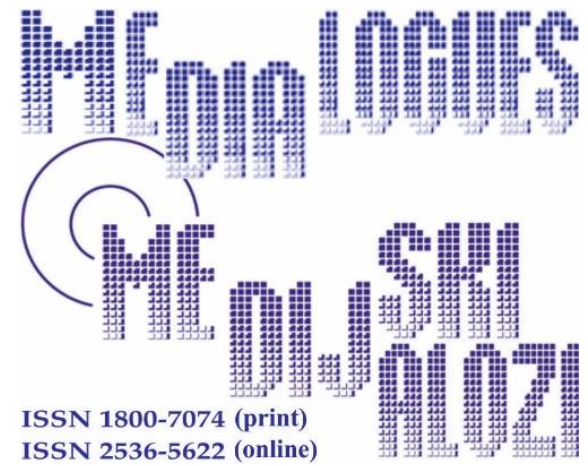

Shilina, M., Delibasic, M. (2011), „Text in the Internet: Non-Classical Trends in Media Research", Media Dialogues / Medijski dijalozi,

Vol. 14, No. 2, pp. 85-95.

\title{
Text in the Internet: Non-Classical Trends in Media Research
}

\author{
Professor MARINA SHILINA, \\ Plekhanov Russian University of Economics, \\ Moscow, Russia
}

Assistant Professor MILICA DELIBASIC,

Mediterranean University, Faculty of Business Studies,

Podgorica, Montenegro

\begin{tabular}{|c|c|}
\hline $\begin{array}{l}A R T I C L E \\
\quad I N F O\end{array}$ & $\begin{aligned} & \text { Received: November 17, } 2021 \text { / Revised from: December 18, } 2021 \\
& \text { Accepted: January 18, } 2021 \text { / } \\
& \text { Available online: April 15, } 2021\end{aligned}$ \\
\hline$D O I$ & $\begin{array}{l}\text { doi.org/10.14254/1800-7074/14-3/6 }\end{array}$ \\
\hline
\end{tabular}

\section{ABSTRACT}

The paper examines the specificity of hypertext as format of information recording on the Internet. Internet hypertext characteristics be-ing unique, the a priori 
co-authorship of the sender and addressee is essential. Analysis of Internet text properties testifies to the emergence of texts of a new type.

KEY WORDS: Internet, hypertext, multimedia, communication model, text type

\section{INTRODUCTION}

Digitalization brings about an ever increasing share of electronic communication formats in the public discourse of the 21 st century. The characteristics of the actual discourse is increasingly defines the Internet ${ }^{1}$. Of these, the Internet is the most massive, running to over 2 billion users worldwide, with 43 million on the $\mathrm{Ru}$ net (according to R. Tagiev's report dated 21.04.2010 - TNS). Communication on the Internet is determined by its unique technological parameters, not to be found in any other communication channel. Principles and models of network communication are a priori fundamentally different from the traditional due to virtuality and interactivity of the WWW. The Web is a system of hypertext documents based on the concept of hypertext, which is based on anthropocentric principles of the creation, transmission, exchange of information, both electronic conventional quasi-verbal text and multime- dia.

The massive emergence and spread of Web hypertext means the emergence of fundamentally new type of text, which requires adequate scientific reflexion. Previous studies, based on the paradigms of hypertext learning and postulates of the theory of classical linear off-line text, do not correspond to the reality of the text on the Internet. In this paper we attempt to study the text in the Internet based on a nonclassical approach, taking into account the current realities of Web communication.

\section{THE INTERNET AS A SPECIFIC MEDIUM}

The Internet is a special form, medium, channel and environment of the social virtual communication. Let us note that, on the Internet, the interaction occurs at the hardware, software and social (Shilina 2007) levels that are interrelated and, together, determine the specific features of Internet communication. The Web's distinctive feature consists in a possibility to directly modify com- munication through the computer network environment from the moment communication is established. We denote the given model as a multisubject one (Shilina 2008).

\footnotetext{
${ }^{1}$ The Internet, a global information computer network providing for the worldwide connection of local networks, gateways, servers and computers using the unified set of rules and protocols which regulate linkage. The World Wide Web, WWW, Web, W3 is the global information space based on the Net's physical infrastructure. The above concepts, though not synonyms, are traditionally used as if they were. In this paper, we used them in the similar way.
} 
The Internet thus projects as an independent subject of social communication, which has never before happened in the communication history. From the standpoint of the communication process as an activity aimed at creation, translation, exchange, multiplication and consumption of an information/text message such transformations denote a cardinal change.

The environment's participation in the communication processes on the Web's latest versions expands, develops and acquires the increasingly complex forms and properties. The present-day software makes it possible to modify communication preventively (e.g. collaborative filtration ${ }^{2}$ ). Online direct interaction ${ }^{3}$ of any number of communication participants in the mode convenient for each of these is a characteristic that is important to the communication-textual activity on the Web. Interactivity implies a possibility of instant feedback, instant increase in the number of senders and provision of their mutual parallel and cross-linked verbal and textual contacts. Open and free access, with the exception of legal traffic restriction in some countries, makes it possible to instantly attract any number of communicators online, which gives rise to the communication processes of all levels of involvement, kinds and formats.

The basic characteristics of Internet communication are being shaped in the online virtual environment. The Internet is a new type of electronic virtuality as compared to other channels such as radio, television, mobile telephony and computer virtuality. The virtual environment creates the "presence effect", i.e. online communicating of the user/addressee involved in his own real environment, in the environment composed of combinations of various electronic computer images generated by multimedia. Virtual presence of a real individual on the Net is particularly attractive to the user/addresser and addressee inasmuch as it enables one to reproduce, transcribe and model any offline situation on the Internet in the required format as well as to form any text strategy. A user may, at that, produce any number of his/her virtual images, which, undoubtedly, affects communication.

The Internet is burgeoning. The new developments are more often designated in the vein of versions: Web 1.0, 2.0, 3.04. The new approach (O'Reilly 2005) was prompted not so much by the need to indicate an advance in software as by the need to change business models of IT companies. From the standpoint of communication, Web 2.0 changes are very important. The communication process is greatly simplified and is very convenient for the common user. This situation stimulates the priority development of personal recourses and communication forms such as blogs, podcasts and videoblogs, makes communication more personalized and emotional and adds the maximum visibility to the user's profile even if this user is anonymous

\footnotetext{
${ }^{2}$ Collaborative filtration, the method providing for filtration relative to user interests basing on previous data on other users' preferences.

${ }^{3}$ We define interactivity as a rightful online polylogue of any number of communicators.

${ }^{4}$ The Web 1.0 designation appeared only after Web 2.0 came into being.
} 
or imaginary. Widespread horizontal homogeneous communication flows form the non-hierarchical discourse unlike the earlier times when communication was hierarchical and unequal/heterogeneous, e.g. programmer-to-user, author-to-reader. Communication has actually become multilateral and multipolar. Alongside the global communication the Web's openness and interactivity promote personalized communication of various kinds, formats and levels. For instance, the most widespread Web 2.0 format is similar to the offline "many-to-many" format. Mass multilateral communication goes over to users' joint interaction. The user masses determine content categorization and the most frequently resort to semantic search, folksonomy, which more accurately reflects the total conceptual model of information requested by the whole user group. Besides, the Internet undergoes change as a collective database: while Web 1.0 mostly featured publication of any heterogeneous content, in the present situation there is the growing amount of requested information ranked through the effort of the "collective mind".

The common user turns into the main active producer of messages, resources, and of their endless transformations. The concept of user-generated content proves pivotal: $70 \%$ of all digital information generated in the world in 2010, according to the International Data Corporation (IDC), were produced by users. Previously an addressee of communication, a user becomes a sender, a subject of Internet communication. E-mergence of the subject-to-subject paradigm (S2S) and model, described by the author, involves a change in the communication trend, conditions and roles as well as com- municator tasks, and this change is basically new for the global public sphere as, in its time, was the Web and the first multisubject model. Such models and the communica- tion process occurring on the Internet are radically different from all those existing offline. Thus Internet communication has some unique properties and, consequently, is a new object of the social communication theory (Shilina 2007, Ibid.). It follows that the Internet studies require new approaches and techniques as well as new theoretic paradigms.

\section{INTERNET HYPERTEXT: FROM GENESIS TO SPECIFICS}

The computer Web-hypertext (Internet hypertext) is the basis for the Web concept as a hypertext documents system and basic format for recording, translation, multiplication and consumption of conventionally verbal text ${ }^{5}$. A hypertext is essentially different from the traditional forms of verbal written texts. Let us look at the specifics of Internet messages. Information on the Web is represented by an Internet hypertext (conventionally verbal written Internet text) and multimedia, which, by format, may be divided into audial (conventionally oral texts, music, etc.) and vi-

\footnotetext{
${ }^{5}$ Text messages on a computer screen are represented in machine languages as 0 and 1.
} 
sual,further subdivided into static (graphics, photo, etc.) and dynamic (video, animation, 3D animation, etc.) information. Synthetic formats are also possible ${ }^{6}$.

Despite its being widespread, an Internet hypertext has not so far been terminologically defined due to its polysemantic nature as a network phenomenon. Hypertext is simultaneously the process of creating a conventionally verbal written text; it is a text itself, be it a singular text or a set of texts; it is a textual paradigm created both by an author and by every reader; it is also a system of associative organization of knowledge in computer networks and a communication mode of a host of individuals. Hypertext is a multilateral communication chain linked with the subject activity of an information/communication producer by the information channel computers, software, and virtual environment, -and by the object-oriented perception, i.e. addressee's co-authorship. Such phenomena impede application of the classical paradigms of text classification and call for some novel nonclassical approaches.

The Encyclopedia Britannica (1994) describes hypertext as a method of linking documents in a network (Subbotin 1994). Hypertext is also defined as a combination of a semantic structure, internal relations of some content structure and computer applications enabling the user to move among the related elements (Conklin 1987). Hypertext is the mechanism of nonlinear relation of text fragments, passages among them (www.urgate.ru) and the principle of data files organization for quick searching (Subbotin Ibid..). M. Subbotin, the first among Russia's researchers to look at the problem, views hypertext as "a combination of a semantic structure, internal relations of some content structure, the technological environment and applications enabling one to master the semantic relations structure and to move among the interrelated elements" (Engelbart 1963).

The specific features of hypertext stem from the underlying concepts, which arose from the first attempts to create new systems of text information structuring ba- sed on associative thinking natural to human mind rather than on formal-logic thin- king. V. Bush's works substantiated the concept of man-oriented information presen- tation and search and, with time, this view turned out to be contrary to the algocentric approach based on the concept of algorithm as a certain computer-oriented sequence of actions.

Potentialities of using computers for the anthropocentric, creative structuring and textual framing of ideas were developed in the 1960s when hyperception shaped primarily by the television and other electronic images became global. In that period, D. Engelbart and other researchers began to view the electronic space of computer communication as the "augment knowledge workshop", which enabled a person to "expand the mind" enhanced the "IQ of man-machine impact" thanks to information

\footnotetext{
${ }^{6}$ Such characteristic is also a ground for an IH classification.
} 
stored in computers, and promoted "the production and reproduction of knowled$g e$ ".

The present-day concept of hypertext as a method of technological support for individual creativity at active work with computer (and later Internet) texts ${ }^{7}$ such as editing or commenting which proved crucial for the Web to be implemented, and the term "hypertext" to designate a computer system's structural unit, was advanced by an American sociologist, philosopher, and pioneer of information technology T. Nelson in early 1960th. In his Xanadu project ${ }^{8}$, he used the ideas of literature as a hypersystem of the interrelated works and computer as an universal system of texts as well as an archive. T. Nelson's idea, remaining viable to this day, was that the computer environment contains a chain of frames in which the associative relations defined by the author generate the three-dimensional information space, which essentially develops as adequate to the information processing trends and generation of ideas by the human brain. According to T. Nelson's (1993) conception, hypertext as an information organization system provides for today's authoring since new knowledge, a new text (a hypertext) is created in conformity with the moves on information searching and processing prescribed by the author.

A hypertext as document expresses the nonlinear structure of ideas created in the process of work with nonlinear documents. The researcher defined hypertext as "non-sequential writing". "Writing" reflects the specificity of a hypertext both as the process of writing and as a new type of text. This definition is not at all contradictory because the concept of hypertext system does not involve the concept of a final document, i.e. a text similar to a linear document recorded on a paper carrier. "Writing" is the process of creation and development of ideas which are the essence of any work and more adequate to the author's conception, and which, ideally, should be perceived by the reader. T. Nelson proposed transclusion, i.e. a possibility of including some texts or text fragments into other texts and references/links between the parts, as the main tool for creating a hypertext and for working with it. A hypertext implied availability of a virtual document, virtual text as an image on the screen; machine languages turn it into a conventionally verbal written form; and movement from reference to reference was supported by the technical means and a vast virtual information base.

The ideas associated with hypertext triggered the development of such computer systems as HyperWave, Microcosm and WebThing in 1980th. The Web system developed by T. Berners-Lee's team became the most widespread, because it "has simplified the data model, has ignored the problems of navigation and integrity of relations. Yet it has made a hypertext popular" (Smith, 1987). Today hypertext is

\footnotetext{
${ }^{7}$ There is also a great number of non-Web hypertext systems. See: Nielsen 1995

${ }^{8}$ Xanadu is the name of the fairy-tale country from S. T. Coleridge's poem "Kubla Khan: Or, A Vision in a dream" which is used to romantically emphasize the "fairy-tale" potentialities of a computer hypertext system.
} 
the basic compo- nent of the Web as a modern computer network using the state-ofthe art software, HTML (hypertext markup language) in particular.

We should discriminate the concepts of computer hypertext $(\mathrm{CH})$ and Internet hypertext (IH). The former is a form of recording the computer data and work with these within the capabilities of a given computer. The idea of hypertext organization of searching and information processing underpinned the Web software which, using computer hypertext and total linkage of computers helped create the unified computer communication space. The technological and, as a consequence, functional differences are a matter of scale (one computer, its software and capacity versus worldwide unification of computers and global access to any software and any information). $\mathrm{CH}$ and $\mathrm{IH}$ represent two basically different communication models, i.e. man-tocomputer and man-to-computer-to-man. The $\mathrm{CH}$ communication model is devoid of the communicative properties offered by the Internet: the environment plays no part in communication modification, and there are no such properties as interactivity, connectivity, sharing common databases and programs, communication optimization, etc. The absence of a co-operation between author and reader, of information multi- dimensionality and impossibility of entering the World Wide Web database are the key differences.

As a computer communication product $\mathrm{IH}$ is a technical method for bringing together information of the Internet database by means of a computer; the technical principle of actualization of the associative relations, transitions, and of the unification of documents into a certain system; a method of functioning for the conventionally verbal written communication on the Web. A hypertext may also be defined as a knowledge organization system in computer networks. In fact, all information on the Internet may be defined as a gigantic hypertext.

\section{INTERNET HYPERTEXT AS CO-AUTHORSHIP COMMUNICATION}

For the linear text's author, transformation of an event/conception into a text traditionally means "its rendition in the system of some or other language, i.e. its subordination to the earlier defined given structural organization... Being rendered by the linguistic means, it is inevitably transferred to the content plane. Thus, the very fact of an event being transformed into a text increases the latter's degree of organization. Moreover, the system of linguistic relations is inevitably transferred onto interpretation of the real-world relations" (Lotman 1992). The activity on IH creation also involves work with ideas realized by choice of the nomination means, semantic structure and (in the case of conventionally verbal written text) syntactic structure, stylistic arsenal and actualization (heading, subheading, lead, ending). At the same time, the classical presentation of the process of author's verbal written text production described above only partially correlates with implementation of a general idea of message in the Internet communication system. 
A hypertext's features and parameters are specific. An author/pro-ducer of information / communication initiates production of a hypertext on the Internet in accordance with his own idea, specific capabilities of a tool (computer) and communication channel (the Internet), then the final hypertext format is shaped in the process of communication by the reader, who de facto becomes a co-author in the final text production.

Reading and perception of a hypertext by the reader/addressee is the activity on reconstruction of the author's hypertext version using references (hyperlinks) to other information sources (texts, multimedia files) included by the author/addresser, and on production of a new text using more references. In other words, to an addressee, a hypertext is authoring a new hypertext. In fact, production and consumption of IH is both authoring and writing and the addressee's authoring may prevail. In the end, the addressee gets, in a sense, his/her own document which indicates the personal preferences and carries the information/message required by this particular addressee, which makes the text significantly more valuable for the addressee. It is also obvious that the reader is extremely loyal to the text he/she personally produced.

It is only logical to refer authorship to the basic parameters of IH: we classify IH as the author's (initial IH) one and the reader's one (final IH) - Shilina, 2007, Ibid. Professional hypertext production starts with determination of a communication goal and target audience, then comes forecasting of variability of text perception by the audience, and a suitable format is chosen. The reader's close following the hypertext author's idea may be interpreted as a demonstration of very high professional qualification of the latter, including the ability to realize the specific communication task.

\section{CHARACTERISTICS OF HYPERTEXT AS AUTHOR'S RESPONSIBILITY}

IH content is to a large extent determined by the Web's technical characteristics, which is another unique feature of the communication process on the Internet and another IH peculiarity. The author/addresser of the initial hypertext and author/ addressee of the final hypertext should have both communication and technical knowledge in order to produce, receive, translate, multiplicate and consume an adequate text message. These kinds of knowledge are needed in order to understand the specifics of the Internet as an independent subject of communication and in order to produce a high-quality hypertext and multimedia message and to include it into the communication process.

The theoretical and practical postulates of IH formation (http: // www.useit.com) are based on the parameters of perception of information by the user on the screen. Let us briefly state the main characteristics of the $\mathrm{IH}$ formation: content is primary; hypertext is more convenient for the user perception if it's laconic (by 53\%), easily browsed (by 47\%), carries information rather than advertising (by 27\%). As to 
content, it is the best when a hypertext is accessible, i.e. the key information can be found quickly; when it is short, i.e. the optimal amount of information can be seen on the screen; when it is well-structured and divided into short paragraphs, with 3-5 paragraphs on the page; when there are subheadings, and content is presented as the "inverted pyramid"; when there are visual markers such as clearly defined initial phra- ses of paragraphs as well as familiar words and concepts; when key words are simple and clear; when there are headings and page titles; when there are hyperlinks under- lined in blue. Photo, audio, video, animation and other multimedia elements should complement the text, making it multidimensional. To index information by search engines, words should belong to the so-called semantic core, reflecting the text's main ideas. To be rated high, the text should be constantly updated. IH is surfed/browsed; the user looks through the text on the screen twice horizontally and then vertically top-to-bottom (the so-called F pattern).

\section{CONCLUSIONS}

The communicological approach to IH presented in this study enables us to make a number of generalizations.

- The communication process on the Internet is basically different from the offline communication in the quantitative and qualitative parameters. In particular, the communication participants are virtual and the environment becomes an independent communication subject. formats.

- On the Internet, information is recorded in the hypertext and multimedia

- IH is a special mechanism and a special form of associative producing and obtaining computer hypertext Internet information by its co-authors - producer and addressee; it is a conventionally verbal written text paradigm, created both by the author-sender and by each of his readers/addressees who are co-authors in their own equal right; it is the process of production and it is a rhizomorphic text; it is a singular text and a host of texts; it is a mode of the author's creative communicati- on with the text and contacts with it of a host of individuals who receive the text.

- Producing, translating, multiplicating and consuming information on the Internet is a process of the joint activity of the author/ addresser and reader/addressee, which forms some basically new parameters of textual communication on the Internet. The subject-to-subject communication model implies that users become the content co-authors, which significantly transforms the Internet hypertext parameters.

- Technologies for production and consumption of IH by the author/ addresser and co-author/addressee are important to the formation of text's conceptual characteristics.

- The communication barriers on the Internet arise at all stages of information gene- ration and consumption and at all communication levels. 
We suggest that there is a typology of threats to the communication process, these including software, programming and social communication threats. The problems are caused by the competence-driven digital divide (Shilina 2009), and by the emergence of prosumers as the basic content producers on the Internet. The development of the nonclassical IH fundamentals system is clearly needed in order to categorize IH. Let us denote the IH typology paradigms (see in greater detail: Ibid.) The- re is no scientific definition for "text type". What there are, are such terms as text type, text genre, discourse type, etc. The text typology advanced by Russia's researchers in recent years (Krivonosov 2001, Ponomarev 2001, Bogoyavlenskiy 2004, Ivanova, 2006) has not so far included computer and Internet texts.

We propose to rely on functionality since it is the technological capabilities of Internet resources that determine the essential characteristics and format of hypertexts used in them. Such a classification may be defined as the most general and horizontal. The genre system of hypertexts may be described as vertical. The genre-forming factors of the traditional verbal texts include " $a$ subject of representation, goal-setting, method of representation, functions and factors of style and language" (Krivonosov Ibid.).

Both the genre-forming and typological factors of IH determine the technological, i.e. functional parameters of resources and companies for which hypertext is produced, and this is another difference of hypertexts from other forms of information recording. We believe that the Internet text genre may be described as a particular form of hypertext material organization that features commonality of the content, structural, compositional, and stylistic attributes. A blog hypertext, for instance, may be described as conventionally verbal textual, monological and convergent (combined with multimedia); a blog notice may be described as a news genre. A site hypertext, in turn, is multi-genre, for it presents different forms of hypertexts.

In the situation where production of hypertext of any type may involve the participation of any reader/addressee as co-author in his own right, there arises an issue of IH institutionality, which is another classification ground. In our view, this ground applies to the professional mass media author's hypertexts, which may be lumped together in this category. On the other hand, thanks to the Internet's unprecedented openness, any text is potentially mass media copy.

The emergence and wide dissemination of a new type of texts on the Internet as a priori media text means building a completely new principles of organization of the text on the Web and professional media: non-linear, multidimensional, multimedia, collaborative, in co-authorship with the active audience, prosumers.

Dentified characteristics of hypertext on the Internet change dramatically classical principles of the textology and the theory and practice of mass media as well. 


\section{BIBLIOGRAPHY}

Bogoyavlenskiy, A. (2004), “ Types of public relations texts and carriers of PR messages ", Vestnik VGU, No. 1 (in Russian).

Conklin, J. (1987), "Hypertext: an Introduction and Survey", Computer, Vol. 20, No. 9, pp. 17-41.

Engelbart, D. C. (1963), A conceptual framework for the augmentation of man's intellect, Vol. 1, D.C. Spartan Books, Washington.

Ivanova, K. (2006), Copywriting: the secrets of composing advertising and PR texts, $\mathrm{SPb}$, Moskva (in Russian).

Krivonosov, A. (2001), PR-text in the public communications system, SPb, Moskva (in Russian).

Lotman, Y. (1992), Semiotics of culture and the concept of text, selected articles, V 3 t. Tallin, T. 1 (in Russian).

Nelson, T. (1993), Literary machines, Mindful Press, Sausalito. CA.

O'Reilly, T. (2005), What is Web 2.0? , http: // oreillynet.com / pub / a / oreilly / tim / news/ 2005 / 09 / 30 / what-is-web-20html.

Ponomarev, S. (2001), "Types of texts in Public Relations", Advisor, No. 4 (in Russian).

Shilina, M. (2007), Corporate Internet resources in the public relations system, Moskva (in Russian).

Shilina, M. (2008), “New communication models on the Internet”,Medi@l'manakh, No. 1 (in Russian).

Shilina, M. (2009), “Media of the 21 century as an object of research”, Medi@ l'manakh, No. 5 (in Russian).

Smith J. B. (1987), The King is Dead; Long Live the King, Hypertext'87, the conference papers.

Subbotin, M. (1994), Hypertext: a new form of written communication. Results of science and technology, T. 18, Informatika-VINITI, Moskva (in Russian).

The New Encyclopedia Britannica (1994), 15th ed., www.urgate.ru http: // www. useit.co. 